\title{
The ART of solving the mystery
}

4

\section{PfKelch 13}

and its

compartment

proteins

regulate endo-

cytosis, which

controls the

amount of

haemoglobin

available for

degradation

and there-

fore the

concentration

of active ART
Delayed treatment of Plasmodium

falciparum can lead to the

progression of severe malaria

and fatal disease. Artemisinin (ART)-based therapies are the global standard for the treatment and control of malaria, but the emergence and spread of ART resistance is a major clinical concern and undermines malaria eradication efforts. Previous studies found that ART resistance manifests during the young ring stage of parasite development in humans, and that ART resistance is conferred by point mutations in the P. falciparum gene Pfkelch13, which encodes a Kelch propeller protein; however, the function and role of PfKelch13 in ART resistance were unknown. Now, Birnbaum, Scharf et al. uncover the molecular mechanism by which mutations in Pfkelch13 render P. falciparum resistant to ART.

Previously, the authors found that PfKelch13 localizes to foci close to the parasite's food vacuole where parasites digest material endocytosed from the host red blood cell - mainly haemoglobin. Notably, digestion of haemoglobin produces haeme, and the interaction of haeme with ART in the food vacuole activates the drug, leading to parasite

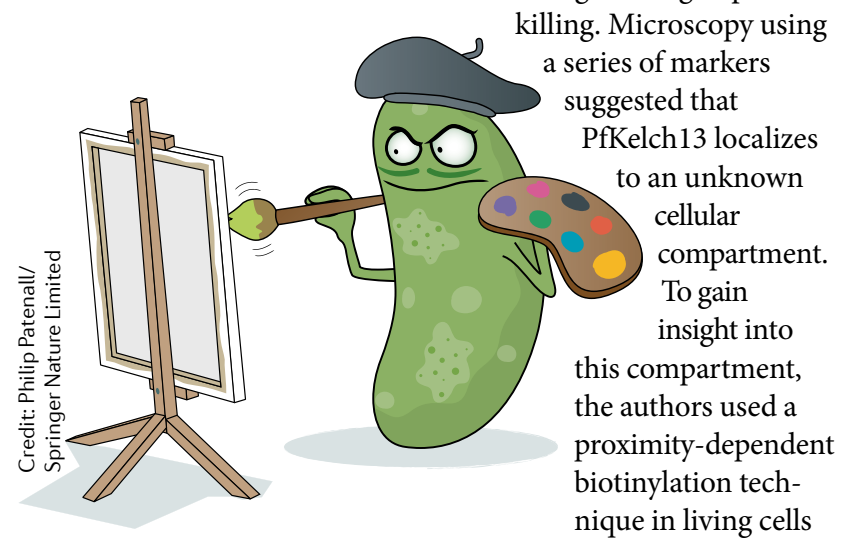

to identify PfKelch13 interactors and proteins within close proximity. A list of high-confidence PfKelch13 interaction candidates (KICs) were identified, including an Eps15-like protein (Eps15) that is involved in endocytosis in other organisms. Eps15 interacted with PfKelch13, which led the authors to hypothesize that the PfKelch13 compartment is involved in endocytosis, the molecular mechanisms of which are poorly understood.

The authors noticed that Eps 15 harboured binding sites for the classic endocytosis clathrin adaptor AP2, which co-localized with PfKelch13, suggesting a role for the PfKelch13 compartment in endocytosis; however, it did not contain clathrin. Microscopy observed Eps15 in close proximity to host cytosol-filled membranous structures within parasites, which PfKelch13 localized to. To directly assess the function of KICs, the authors conditionally inactivated the expression of KIC genes and assayed parasite growth and haemoglobin uptake and found that UBP1, KIC7, Eps15 and AP2 all have an important role in transport of haemoglobin into the food vacuole, but PfKelch13 itself was only necessary for haemoglobin uptake during ring-stage development.

Next, the authors investigated whether an ART-resistant parasite carrying the naturally occurring PfKelch13 ${ }^{\mathrm{C} 580 \mathrm{Y}}$ mutation could endocytose haemoglobin and found that the ART-resistant parasite displayed reduced endocytic uptake, suggesting that this is the reason for reduced susceptibility of ART. Additionally, when PfKelch13 was inactivated, a reduction in endocytosis and
ART resistance levels comparable to PfKelch $13^{\mathrm{C} 580 \mathrm{Y}}$ were observed. From these observations, the authors concluded that early ring-stage parasites endocytose haemoglobin, which is reduced in inactivated PfKelch13 or resistance-conferring mutants, and that this reduction correlates with ART resistance.

After establishing a relationship between the Pfkelch13 mutation and ART resistance, the authors assessed the role of KICs in ART resistance. When conditionally inactivated, all Kelch13 compartment proteins involved in endocytosis rendered parasites resistant to ART, which suggests that Kelch13 compartment proteins are members of an ART resistance pathway, and that a reduction in endocytosis is the mechanism of ART resistance.

The authors found that reduced activity of PfKelch13 is the most likely mechanism of how PfKelch13 mutations cause resistance. A reduction in function also explains why inactivation of PfKelch13 compartment proteins renders parasites resistant, as they are members of a common pathway.

Altogether, these findings led the authors to propose a model whereby PfKelch13 and its compartment proteins regulate endocytosis, which controls the amount of haemoglobin available for degradation and therefore the concentration of active ART. Importantly, these findings may lead to new approaches to overcome ART resistance.

Ashley York

ORIGINAL ARTICLE Birnbaum, J., Scharf, S. et al. A Kelch13-defined endocytosis pathway mediates artemisinin resistance in malaria parasites. Science 367, 51-59 (2020) 